\section{Erhöhtes Krebsrisiko in der Familie von Nichtrauchern}

\author{
Aus populationsbasierten Studien gibt es Hinweise auf eine \\ mögliche genetisch basierte Suszeptibilität für Lungenkrebs, \\ die für eine familiäre Häufung der Erkrankung bei \\ Nichtrauchern verantwortlich sein soll. Dem möglichen \\ Zusammenhang zwischen familiärer Anamnese und dem \\ Lungenkrebsrisiko bei Nichtrauchern sind $\mathrm{H}$. Lin et al. jetzt \\ gezielt nachgegangen. \\ Lung Cancer 2015; 89: 94-98
}

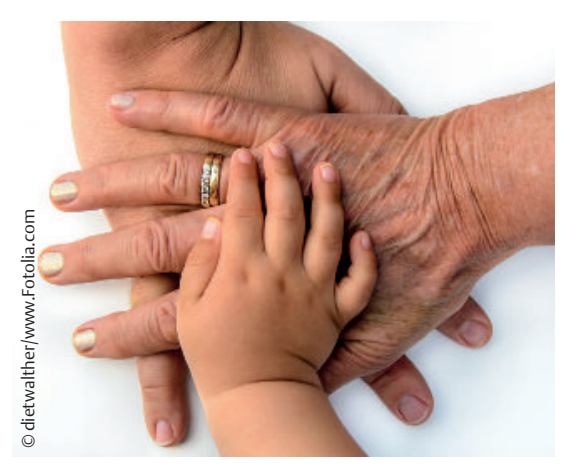

Die Wissenschaftler führten hierzu eine Fall-Kontroll-Studie mit 309 Patienten mit Lungenkrebs und 509 Kontrollen durch, ketztere waren Ehepartner von Patienten und Angehörige, die wie die Patienten selbst angaben, nie geraucht zu haben. Mit Hilfe eines strukturierten Fragebogens erhoben die Autoren neben demografischen Daten (Alter, Geschlecht, Abstammung) auch Daten zu Passivrauch-Exposition, Kochgewohnheiten, Belüftung im Haushalt, beruflicher Exposition und der industriellen Schadstoffbelastung am Wohnort oder Arbeitsplatz. Zur Identifikation von Assoziationen der familiär bedingten Krebswahrscheinlichkeit adjustierten sie die multiple logistische Regressionsanalyse um diese Faktoren.

$30 \%$ aller Patienten waren Passivraucher. 266 der 318 Patienten litten unter einem Adenokarzinom. Mehr Fälle als Kontrollen wiesen anamnestisch eine Lungenerkrankung auf (29 vs. $8 \%$ ). Die Schadstoffexposition im Haushalt und bei der Arbeit war in beiden Gruppen vergleichbar. Die Lungenkarzinom-Patienten hatten aber häufiger einen erstgradigen Famili- enangehörigen, der bereits an Krebs erkrankt war, als die Kontrollpersonen (34,6 vs. 22,8\%). Das galt insbesondere für die Mütter der Patienten (adjustierte Odds Ratio [aOR] 2,64, p<0,001).

Die familiäre Lungenkrebsanamnese war ein starker Prädiktor für das eigene Krebsrisiko (aOR 3,21; p<0,001) wie auch für andere Krebserkrankungen (aOR 1,79; $\mathrm{p}<0,001)$. Die weitere Analyse anderer von einer Krebserkrankung betroffenen Familienmitglieder zeigte einen Geschlechtsunterschied: Ein männlicher erstgradiger Verwandter des nicht rauchenden Lungenkrebspatienten hatte ein um 54\% erhöhtes allgemeines Krebsrisiko im Vergleich zu Angehörigen von Kontrollen ein. Das Risiko für ein Bronchialkarzinom für diese Verwandten stieg um den Faktor 2,25 an. Bei erstgradigen weiblichen Verwandten des Lungenkrebspatienten war das allgemeine Krebsrisiko um den Faktor 2,37 und das Lungenkrebsrisiko um den Faktor 7,31 erhöht.

Fazit
Die Fall-Kontroll-Studie fügt der beste-
henden Evidenz für genetische Faktoren
beim Bronchialkarzinom von Nichtrau-
chern weitere Belege hinzu. Besonders
ausgeprägt ist der Einfluss solcher Fak-
toren bei einer mütterlichen Krebser-
krankung. Ob das erhöhte Krebsrisiko
der weiblichen erstgradigen Verwand-
ten auf eine besondere Suszeptibilität
für Karzinogene hinweist, oder auf an-
dere bisher nicht bekannte Risikofakto-
ren zurückzuführen ist, ist nach Anga-
ben der Autoren unklar.

Friederike Klein, München
Onkologie

\section{Lungenkarzinom besänftigt das Immunsystem}

Bestimmte Lungentumoren machen sich einen natürlichen Schutzmechanismus aus der Schwangerschaft zunutze. Das nicht-kleinzellige Lungenkarzinom (NSCLC) sowie seine Absiedlungen in anderen Organen schütten bei Frauen und Männern Glycodelin aus und unterdrücken damit wahrscheinlich in ihrer unmittelbaren Umgebung Abwehrreaktionen des Immunsystems. Das Protein ist im Blut nachweisbar und könnte sich als Biomarker für die Früherkennung und Verlaufskontrolle eignen. Die Ergebnisse der Heidelberger Studie sind jüngst in der Zeitschrift Clinical Cancer Research (DOI: 10.1158/10780432.CCR-14-2464) erschienen. Die Forscher vermuten, dass die Krebszellen Glycodelin ausschütten, um Immunzellen in ihrer direkten Umgebung zu betäuben. Diese können so keine Abwehrreaktion einleiten. Hinderten die Wissenschaftler die Tumorzellen im Labor daran, das Protein herzustellen, bildeten diese stattdessen andere Proteine, die das Immunsystem beeinflussen. Sie verglichen außerdem den Glycodelin-Spiegel in konservierten Blutproben von mehr als 25 Patienten mit deren Krankheitsverlauf. Die Konzentration von Glycodelin im Blut korrelierte sehr gut mit dem Therapieansprechen oder dem Fortschreiten der Erkrankung. Schlug eine Chemotherapie an oder wurde der Tumor entfernt, sank der Glycodelinspiegel. Wuchs der Tumor weiter oder bildeten sich im späteren Verlauf Absiedlungen, stieg die Konzentration an. Bestätigt sich dieser Zusammenhang in weiteren, bereits angelaufenen Studien, könnte das Protein als leicht zu testender Biomarker in der Früherkennung und Verlaufskontrolle speziell beim NSCLC eingesetzt werden. Regelmäßige Kontrollen des Glycodelin-Spiegels könnten zusammen mit weiteren Untersuchungen den Ärzten dabei helfen, auf ein Fortschreiten der Erkrankung schnell zu reagieren und die Therapie anzupassen. In künftigen Studien sollte außerdem geklärt werden, ob Glycodelin sich als mögliches Ziel für die Therapie beim NSCLC eignet.

Nach einer Mitteilung des Universitätsklikums Heidelberg 